\title{
Wogonoside induces autophagy-related apoptosis in human glioblastoma cells
}

\author{
LI ZHANG, HANDONG WANG, ZIXIANG CONG, JIANGUO XU, \\ JIANHONG ZHU, XIANGJUN JI and KE DING \\ Department of Neurosurgery, Jinling Hospital, School of Medicine, Nanjing University, \\ Nanjing, Jiangsu 210002, P.R. China
}

Received April 22, 2014; Accepted June 10, 2014

DOI: $10.3892 /$ or.2014.3294

\begin{abstract}
Wogonoside, a bioactive flavonoid extracted from the root of Scutellaria baicalensis Georgi, has shown preclinical anticancer efficacy in various cancer models. However, the effects of wogonoside on glioblastoma cells remain unclear. In the present study, we found that wogonoside exhibited a cytotoxic effect on human glioblastoma cells. The suppression of cell viability was due to the induction of mitochondrial apoptosis. Furthermore, the presence of autophagic hallmarks, including an increase in punctate microtubule associated protein 1 light chain 3 (LC3) dots, changes in cellular morphology and increased levels of autophagy-related proteins were observed in the wogonosidetreated cells. Wogonoside treatment also enhanced autophagic flux as reflected by the increased acidic vesicular organelle (AVO) formation, p62 degradation and LC3 turnover. Notably, blockade of autophagy by a chemical inhibitor or RNA interference decreased the anticancer effect of wogonoside. In addition, the p38 mitogen-activated protein kinase (MAPK) signaling pathway, the phosphatidylinositide 3-kinase/protein kinase B/mammalian target of rapamycin/p70S6 kinase (PI3K/ AKT/mTOR/p70S6K) signaling pathway and reactive oxygen species (ROS) participated in wogonoside-induced autophagy and apoptosis. These findings support the initiation of further studies of wogonoside as a candidate for the treatment of human malignant glioma.
\end{abstract}

\section{Introduction}

Glioblastoma multiforme (GBM) is the most common and lethal primary brain tumor in adults (1). Despite decades of

Correspondence to: Professor Handong Wang, Department of Neurosurgery, Jinling Hospital, 305 East Zhongshan Road, Nanjing, Jiangsu 210002, P.R. China

E-mail: njhdwang@hotmail.com

Key words: wogonoside, autophagy, glioblastoma, mitochondrial apoptosis, reactive oxygen species, p38 mitogen-activated protein kinase, phosphatidylinositide 3-kinase/protein kinase B/mammalian target of rapamycin/p70S6 kinase signaling pathway efforts and advances in surgery, radiotherapy and chemotherapy, the prognosis of patients with GBM remains poor with an estimated median survival of less than 1 year $(2,3)$. Therefore, exploration of a new approach is urgently needed.

Scutellaria baicalensis Georgi (Huang Qin) is one of the most popular herbs in traditional Chinese medicinal diet (CMD) (4). Wogonoside is a bioactive flavonoid extracted from the root of Scutellaria baicalensis Georgi. This drug was originally developed as an anti-inflammatory agent $(5,6)$. However wogonoside has also recently shown preclinical anticancer efficacy in various cancer models, including those of breast cancer (7), bladder cancer (8) and hematopoietic malignancies $(9,10)$. However, the mechanisms by which wogonoside kills cancer cells remain unclear. Several mechanisms such as inhibition of mTOR activity, activation of MAPK and phospholipid scramblase 1 (PLSCR1) $(7,9)$ have been proposed to account for the cytotoxicity of wogonoside.

Apoptosis and autophagy are two major routes of programmed cell death (PCD). Apoptosis (type I PCD) is a highly controlled process involving well-characterized morphological changes, including cell volume loss, chromatic condensation and nuclear fragmentation (11). Autophagy is a lysosomal degradation pathway that degrades damaged organelles into basic biomolecules (12). Under stress situations such as nutrient depletion, autophagy provides the nutriments required to maintain the metabolism essential for cell survival (13). However, extensive activation of autophagy can lead to cell death (type II PCD). Recent studies have shown that various agents known to induce apoptosis also activate autophagy. However, the relationship between autophagy and apoptosis is intricate. Inhibition of autophagy can enhance the apoptosis induced by cytotoxic agents $(14,15)$. In these cases, autophagy has a protective role. However, in other cases, blockade of autophagy attenuates apoptosis $(16,17)$. The discrepancies may be due to the complex and diverse interplays between autophagy and apoptosis. Depending on cell type, stimulus and environment, autophagy and apoptosis may have additive, inhibitory or even synergistic effects.

In the present study, we demonstrated that wogonoside induced autophagy and subsequent apoptosis in human glioblastoma cells. Inhibition of autophagy decreased wogonoside-induced apoptosis. Importantly, we found that the p38 MAPK signaling pathway, PI3K/AKT/mTOR/p70S6K 
signaling pathway and ROS played an important role in the regulation of autophagy and apoptosis.

\section{Materials and methods}

Cell culture. Primary mouse cortical neurons were prepared as described previously (18). In brief, cortical neuronal cells were isolated fromembryos of time-mated pregnant mice and cultured onto poly-D-lysine-coated 96-well dishes at a density of $2 \times 10^{4}$ cells/well in neurobasal medium (Life Technologies, USA) supplemented with 2\% B27 (Life Technologies) and $1 \mathrm{mM}$ glutamate (G3291; Sigma-Aldrich). Cells were maintained in growth medium at $37^{\circ} \mathrm{C}$ in $5 \% \mathrm{CO}_{2}$. Half of the culture medium was replaced with fresh medium every 3 days. All experiments were performed after 10-12 days in vitro. Human U251MG, U87MG and A172 glioblastoma cell lines were obtained from the American Tissue Culture Collection (ATCC; Manassas, VA, USA) and cultured in Dulbecco's modified Eagle's medium (DMEM; Life Technologies, USA) with $10 \%$ fetal bovine serum (FBS; 10099141, Life Technologies) and $1 \%$ penicillin/streptomycin at $37^{\circ} \mathrm{C}$ in a $5 \% \mathrm{CO}_{2}$ incubator. The human SHG44 glioblastoma cell line originally present in our laboratory was cultured in RPMI-1640 (Life Technologies) medium with $10 \%$ FBS and $1 \%$ penicillin/streptomycin at $37^{\circ} \mathrm{C}$ in a $5 \% \mathrm{CO}_{2}$ incubator.

Reagents and antibodies. Wogonoside was purchased from Shanghai Tauto Biotech Co., Ltd. (Shanghai, China). U0126, SB203580, SP600125, LY294002, 3-methyladenine (3-MA), bafilomycin A (BafA1), N-acetyl-cysteine (NAC) and acridine orange $(\mathrm{AO})$ were purchased from Sigma-Aldrich (St. Louis, MO, USA). Z-VAD-FMK (Z-VAD) was purchased from Abcam (Cambridge, MA, USA). Anti-LC3 and anti-Beclin 1 antibodies were purchased from Novus Biologicals (Littleton, CO, USA). Anti-cytochrome $c$, anti-p62/SQSTM1 and anti-Bcl-2-associated X protein (Bax) antibodies were purchased from Abcam. Anti-phosphorylated extracellular regulated protein kinase (ERK), anti-ERK, anti-phosphorylated c-Jun N-terminal kinase (p-JNK), anti-JNK, anti-phosphorylated p38, anti-p38, anti-phosphorylated AKT, anti-AKT, anti-phosphorylated mTOR, anti-mTOR, anti-phosphorylated p70S6K, anti-p70S6K, anti-poly(ADPribose) polymerase (PARP), anti-caspase-3, anti-caspase-8, anti-autophagy-related 3 (Atg3), anti-autophagy-related 5 (Atg5), anti-autophagy-related 7 (Atg7), anti-autophagyrelated 12 (Atg12), anti-B-cell lymphoma 2 (Bcl-2), anti- $\beta$-actin and goat anti-rabbit IgG $(\mathrm{H}+\mathrm{L})$-HRP secondary antibodies were purchased from Cell Signaling Technology (Danvers, MA, USA).

Cell viability assay. Cell viability was assessed by the CCK- 8 assay (Beyotime, Shanghai, China). For primary mouse cortical neurons, $2 \times 10^{4}$ cells/well were seeded onto 96 -well culture plates and cultivated for 10-12 days to adhere. For glioblastoma cells, $5 \times 10^{3}$ cells/well were seeded onto 96-well culture plates and cultivated for $24 \mathrm{~h}$ to adhere. After treatment, CCK-8 (10 $\mu \mathrm{l})$ was added into every well and incubated for $2 \mathrm{~h}$, and the OD value was read at $450 \mathrm{~nm}$ using a Bio-Rad enzyme linked immunosorbent assay (ELISA) microplate reader (Bio-Rad Laboratories, Hercules, CA, USA). The following formula was used: Cell viability $=(\mathrm{OD}$ of the experimental sample/OD of the control group) $\mathrm{x} 100 \%$.

Western blotting. After treatment, cells were harvested and lysed. An equal amount of protein was separated by sodium dodecyl sulfate-polyacrylamide gel electrophoresis (SDS-PAGE, 10\%) and transferred to polyvinylidene fluoride (PVDF) membranes. After blocking with 5\% non-fat dried milk for $2 \mathrm{~h}$, the membrane was incubated with the primary antibodies overnight at $4^{\circ} \mathrm{C}$. Then the immunoreactive bands were visualized by enhanced chemiluminescence using HRP-conjugated IgG secondary antibodies.

Immunofluorescence. Treated cells were fixed using $4 \%$ paraformaldehyde, permeabilized with $0.1 \%$ Triton X-100 and then blocked with immunostaining blocking buffer (Beyotime, Shanghai, China). Subsequently, cells were incubated with the LC3 antibody overnight at $4^{\circ} \mathrm{C}$. Following washes with phosphate-buffered saline (PBS), the coverslips were incubated with a red-labeled secondary antibody (Beijing Zhongshan Jinqiao Biotechnology Co., Ltd., Beijing, China) for $4 \mathrm{~h}$ at room temperature. Nuclei were counterstained with 4',6-diamidino-2-phenylindole (DAPI) and then cells were washed with PBS and mounted with anti-fade medium. The staining was examined using a fluorescence microscope.

Flow cytometric (FCM) analysis of apoptosis. After treatment, cells were washed twice with PBS and collected by trypsinization. After centrifugation at $1000 \mathrm{x}$ g for $5 \mathrm{~min}$ at room temperature, the cells were re-suspended and stained with Annexin V and propidium iodide (PI) (BD Biosciences, San Jose, CA, USA) for $15 \mathrm{~min}$ at room temperature in the dark. Flow cytometry was performed using a FACScan laser flow cytometer (FACSCalibur; Becton Dickinson). Then the apoptotic rate was analyzed by the FACScan laser flow cytometer (FACSCalibur).

Measurement of the formation of acidic vesicular organelles (AVOs). Treated cells were stained with $1 \mu \mathrm{g} / \mathrm{ml}$ AO for $15 \mathrm{~min}$ at $37^{\circ} \mathrm{C}$. After incubation, cells were washed three times with PBS and then observed immediately under a fluorescence microscope.

Ultrastructural analysis of autophagy by transmission electron microscopy (TEM). After treatment, cells were collected by trypsinization, washed three times with warm PBS $\left(37^{\circ} \mathrm{C}\right)$ and then fixed for $1 \mathrm{~h}$ in $2.5 \%$ glutaraldehyde in $0.1 \mathrm{M}$ cacodylate buffer with $1 \%$ sucrose. After washing in PBS, cells were then embedded, sectioned, double stained with uranyl acetate and lead citrate (Fluka, St. Louis, MO, USA) and viewed using a JEOL JEM-1011 TEM (Tokyo, Japan).

RNA interference with shRNA. The lentiviral vectors were purchased from GenePharma Co., Ltd. (Shanghai, China). Lentiviral vectors for expression of scrambled or Beclin 1 shRNA were diluted in medium containing $5 \mu \mathrm{g} / \mathrm{ml}$ Polybrene. The shRNAs were then added to the cultures. After $72 \mathrm{~h}$, the transfected cells were selected using puromycin $(5 \mu \mathrm{g} / \mathrm{ml})$ for $24 \mathrm{~h}$. The human Beclin 1 shRNA sequence was 5'-GAA TGT CAG AAC TAC AAA CGC TGT T-3', while the scrambled 
A

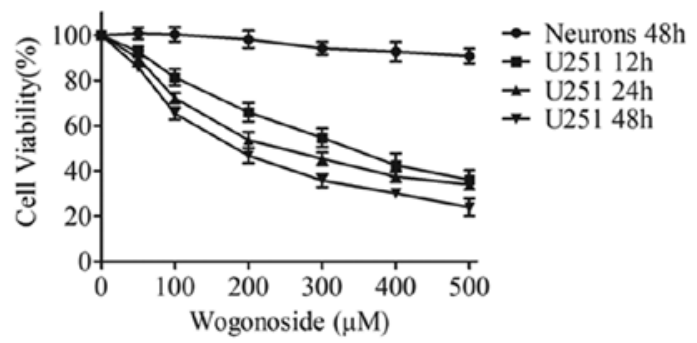

$\mathrm{C}$

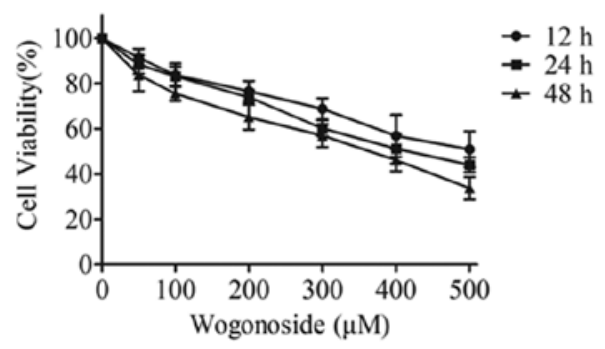

B

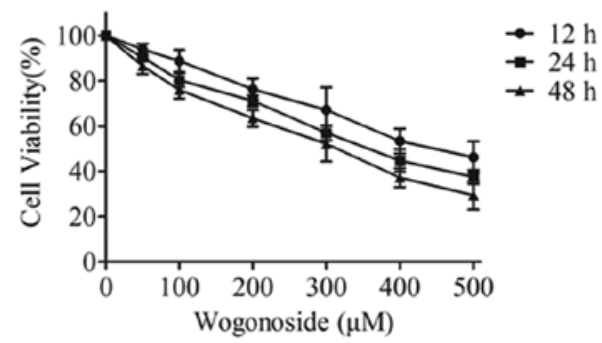

D

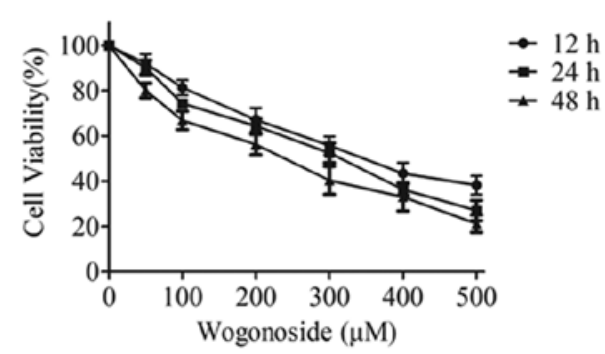

Figure 1. Wogonoside induces cell death in human glioblastoma cells. (A-D) Human glioblastoma cell lines U251MG, A172, SHG44, U87MG and mouse primary astrocytes were treated with wogonoside at the indicated concentrations for different time periods, and the cell viability was analyzed by CCK-8 assay. All values represent the means \pm SD of three independent experiments.

shRNA sequence was 5'-TTC TCC GAA CGT GTC ACG T-3'. Western blotting was performed to validate the knockdown efficiency, and cells were split for the different assays.

Statistical analysis. The SPSS19.0 software package was used to perform all statistical analyses. Data are expressed as means \pm SD. The statistical significance of the differences between treatments was assessed using one-way ANOVA followed by Student-Neuman-Keuls test for multiple comparisons. A probability $(\mathrm{P})$ value $<0.05$ was considered to indicate a statistically significant result.

\section{Results}

Wogonoside inhibits cell viability and induces mitochondrial apoptosis in glioblastoma cells. To examine the effect of wogonoside on cell viability, four human glioblastoma cell lines, U251MG, SHG44, A172 and U87MG, were treated with different concentrations of wogonoside for different time periods. Cell viability assays showed that wogonoside induced cell death in both a concentration- and time-dependent manner in all the cell lines tested (Fig. 1). The $\mathrm{IC}_{50}$ values of these cell lines are shown in Table I. Importantly, wogonoside alone did not affect the cell viability of the mouse primary neurons (Fig. 1A). Since U251MG was the most sensitive cell line in respond to wogonoside, this cell line was chosen for the subsequent studies unless otherwise indicated.

To determine whether the reduced cell viability was due to apoptosis, flow cytometric analysis for Annexin V/PI was used. As shown in Fig. 2A, a significant increase in the apoptotic population was observed in cells treated with wogonoside. In addition, wogonoside cleaved caspase-3 and PARP, downregulated $\mathrm{Bcl}-2$, upregulated $\mathrm{Bax}$ and released cytochrome $c$ from the mitochondria without affecting caspase-8 (Fig. 2B and C),
Table I. IC $_{50}$ values of wogonoside in the human glioblastoma cell lines.

\begin{tabular}{lllll}
\hline & \multicolumn{4}{c}{$\mathrm{IC}_{50}(\mu \mathrm{mol} / \mathrm{l})$} \\
\cline { 2 - 5 } Cell lines & $\mathrm{U} 251 \mathrm{MG}$ & $\mathrm{SHG} 44$ & $\mathrm{~A} 172$ & $\mathrm{U} 87 \mathrm{MG}$ \\
\hline Time (h) & & & & \\
12 & 335.03 & 522.29 & 523.46 & 340.64 \\
24 & 248.39 & 409.40 & 348.57 & 274.30 \\
48 & 193.44 & 305.08 & 269.54 & 199.83 \\
\hline
\end{tabular}

$\mathrm{IC}_{50}$ values for each cell lines were calculated using Compusyn software.

suggesting that mitochondrial apoptosis was induced by wogonoside. To further demonstrate that wogonoside induced caspase-dependent apoptosis, cells were co-treated with wogonoside and the pan-caspase inhibitor Z-VAD, and the cell viability as well as caspase-3 and PARP cleavage were determined. The results revealed that Z-VAD treatment suppressed cell death and caspase-3 and PARP cleavage induced by wogonoside (Fig. 2D and E). Collectively, these data indicate that wogonoside induced cell death in human glioblastoma cells through the mitochondrial apoptosis pathway.

Wogonoside induces autophagy in glioblastoma cells. A previous study showed that wogonoside activates autophagy in breast cancer cells (7). Thus, we aimed to ascertain whether autophagy occurs in the cells treated with wogonoside. We first used immunofluorescence to clarify the ability of wogonoside to induce autophagy. Results of the fluorescence microscopy revealed that wogonoside induced the formation 
A
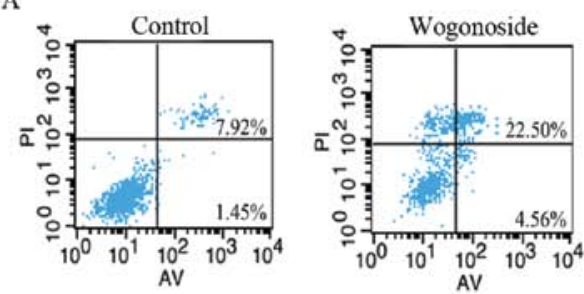

$\mathrm{D}$

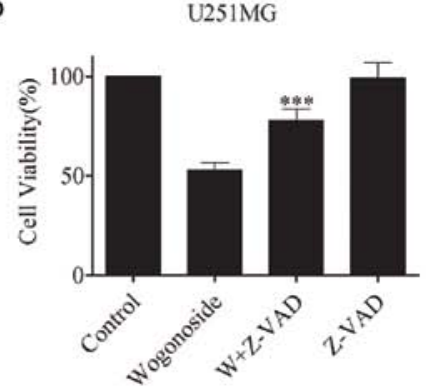

B

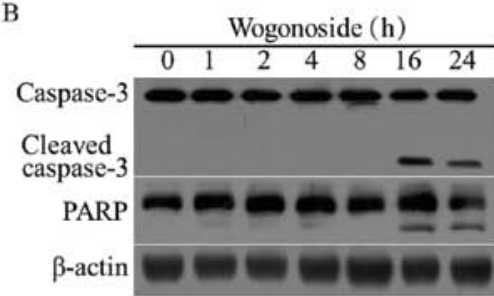

$\mathrm{E}$

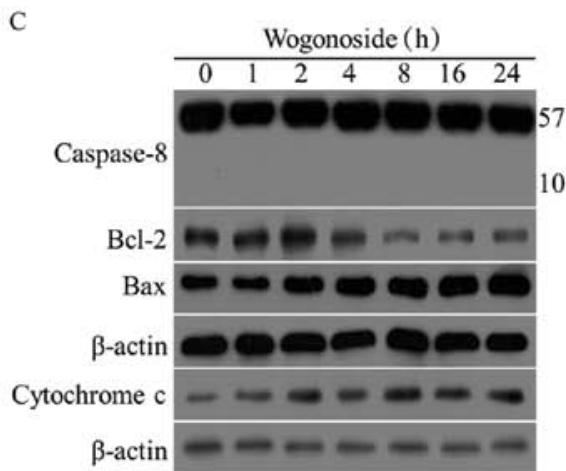

Figure 2. Wogonoside induces mitochondrial apoptosis in the U251MG cells. (A) Cells were treated with DMSO or $250 \mu \mathrm{M}$ wogonoside for $24 \mathrm{~h}$, and apoptosis was determined by flow cytometry followed by Annexin V/PI staining. (B and C) Cells were treated with $250 \mu \mathrm{M}$ wogonoside for different time periods and the expression of caspase-3, PARP, caspase- $8, \mathrm{Bcl}-2$, Bax and cytochrome $c$ was detected by western blotting. (D and E) Cells were pre-treated with $25 \mu \mathrm{M}$ Z-VAD for $1 \mathrm{~h}$ followed by the treatment of $250 \mu \mathrm{M}$ wogonoside for another $24 \mathrm{~h}$. The cell viability (D) and cleavage of caspase-3 and PARP (E) were analyzed. Columns in histogram; mean of three independent experiments; bars; SD. ${ }^{* * *} \mathrm{P}<0.001$ vs. cells treated with wogonoside alone. $\beta$-actin was used as loading control. DMSO, dimethyl sulfoxide.

of autophagosomes, as indicated by the formation of LC3 punctate dots in the cells (Fig. 3A). Furthermore, TEM analysis showing autophagic vacuoles containing cellular material or membranous structures further confirmed the presence of autophagy (Fig. 3B). Meanwhile, wogonoside treatment increased the expression levels of Beclin 1, LC3-II and various other autophagy-related proteins in a time-dependent manner (Fig. 3C).

Induction of autophagy includes not only an increase in autophagosomes, but also an upregulation of autophagic flux. To examine the autophagic flux, we first analyzed the formation of AVOs by AO staining. As shown in Fig. 3D, treatment with wogonoside resulted in the formation of red fluorescent AVOs. During the process of autophagy, p62 is wrapped into autophagosomes and degraded in autolysosomes. Thus, we determined the protein levels of p62 in the wogonosidetreated cells. Results of the western blot analysis showed that wogonoside reduced the amount of p62 in a time-dependent manner (Fig. 3E). We further examined the autophagic flux by LC3 turnover assay. Treatment with BafA1, which blocks autolysosome formation, led to a significant increase in LC3-II levels. The BafA1-induced increase in LC3-II was augmented when combined with wogonoside (Fig. 3F). In line with the enhanced LC3 turnover, the wogonoside-induced reduction in p62 was prevented by BafA1 (Fig. 3F). Together, these data iindicate that wogonoside induced autophagy in glioblastoma cells.

Autophagy is required for wogonoside-induced apoptosis. We next aimed to elucidate the role of autophagy in wogonoside-induced apoptosis. To this end, we first used
3-MA, an inhibitor that blocks the autophagy pathway at early stages. As shown in Fig. 4A-C, 3-MA reversed not only autophagy but also the cell death and apoptosis caused by wogonoside. To exclude the off-target effects of 3-MA, we used shRNA to specifically knock down Beclin 1. Fig. 4D shows that the levels of Beclin 1 and LC3-II were apparently decreased in the Beclin 1 shRNA-treated cells. In addition, knockdown of Beclin 1 decreased cell death and cleavage of PARP (Fig. 4E and F). Finally, we used flow cytometry to determine the role of autophagy in apoptosis. As shown in Fig. 4G, both 3-MA and knockdown of Beclin 1 reduced the apoptosis in response to wogonoside. Collectively, these data strongly suggest that wogonoside-induced autophagy is a prerequisite to cell apoptosis.

Wogonoside activates the p38 MAPK signaling pathway and inhibits the PI3K/AKT/mTOR/p70S6K signaling pathway. It has been well documented that the MAPK and $\mathrm{PI} 3 \mathrm{~K} / \mathrm{AKT} / \mathrm{mTOR} / \mathrm{p} 70 \mathrm{~S} 6 \mathrm{~K}$ signaling pathways play an important role in regulating both autophagy and apoptosis. Therefore, the effects of wogonoside on the activity of these two pathways were studied. Fig. 5A and B showed that wogonoside treatment significantly increased the phosphorylation of p38 and decreased the phosphorylation of AKT, mTOR and p70S6K in a time-dependent manner. These results suggest that p38 MAPK and PI3K/AKT/mTOR/p70S6K signaling pathways may participate in the autophagy and apoptosis induced by wogonoside. To confirm this, we assessed the effects of a number of pharmacologic inhibitors, including those of MEK1/2 kinase (U0126), JNK kinase (SP600125), p38 kinase (SB203580) and PI3K kinase (LY294002) on the cell death induced by wogonoside. Of 
A

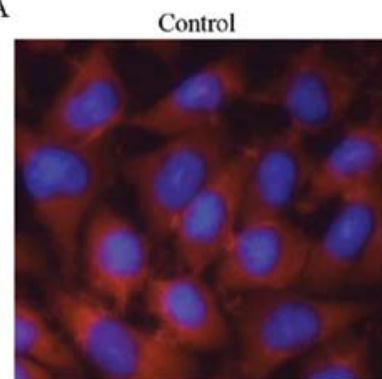

B

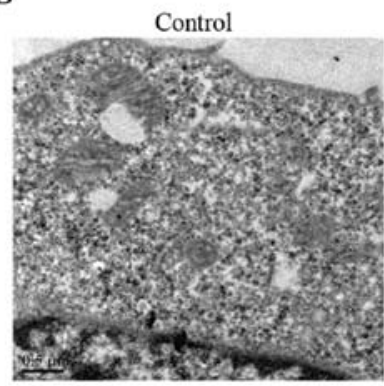

Control

D

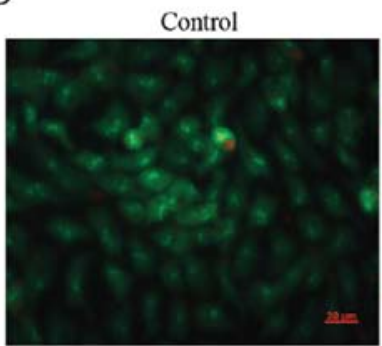

Wogonoside

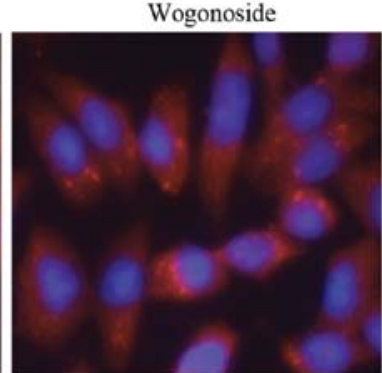

Wogonoside

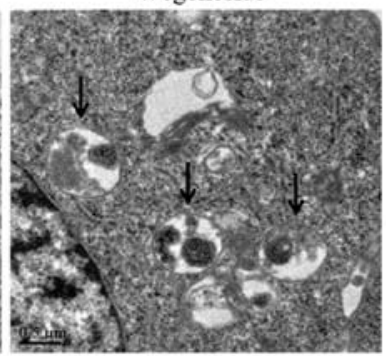

$\mathrm{E}$

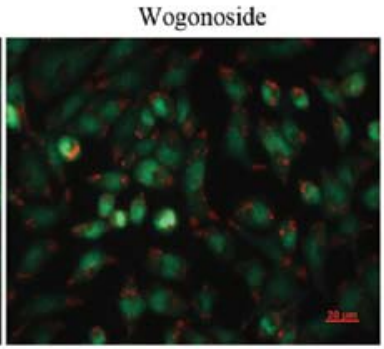

F
U25IMG
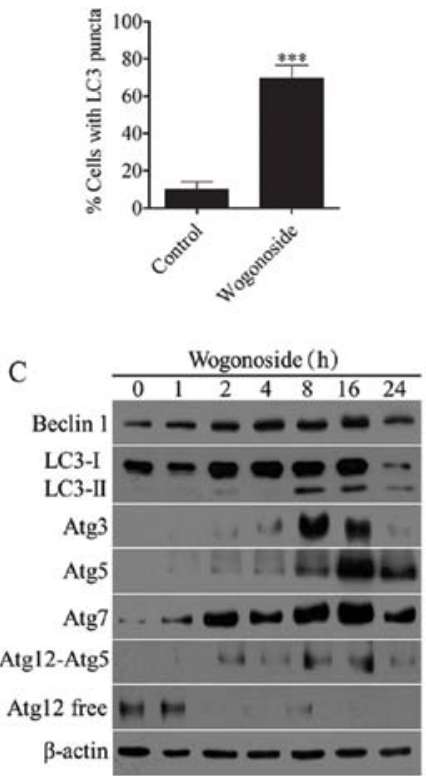

E
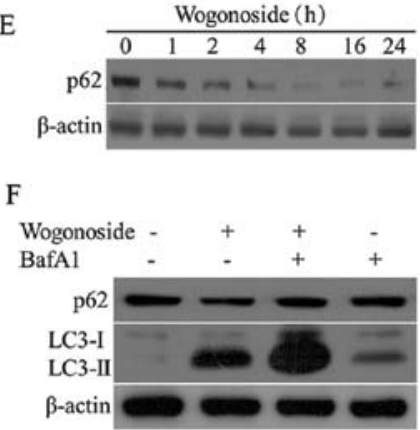

Figure 3. Wogonoside induces autophagy in U251MG cells. (A) Cells were treated with DMSO or $250 \mu \mathrm{M}$ wogonoside for $24 \mathrm{~h}$, and stained with the LC3 antibody and appropriate secondary antibody. Cells were then observed under a fluorescence microscope. The nuclei were stained with DAPI. The percentage of cells presenting typical LC3 puncta ( $>5$ puncta/cell) is shown. Data are means \pm SD from three independent experiments (at least 100 cells were counted for each experiment; ${ }^{* * *} \mathrm{P}<0.001$ ). (B) Cells were treated with DMSO or $250 \mu \mathrm{M}$ wogonoside for $24 \mathrm{~h}$, then harvested and subjected to transmission electron microscopy as described in Materials and methods. Black arrows indicate autophagosomes including residual digested organelles. Scale bar; $0.5 \mu \mathrm{m}$. (C) After cells were exposed to $250 \mu \mathrm{M}$ wogonoside for various time periods, cell lysates were subjected to western blotting. (D) Cells were treated with DMSO or $250 \mu \mathrm{M}$ wogonoside for $24 \mathrm{~h}$. After treatment, cells were stained with AO for $15 \mathrm{~min}$ and observed under a fluorescence microscope. Scale bar; $20 \mu \mathrm{m}$. (E) Cells were treated with $250 \mu \mathrm{M}$ wogonoside for various time periods, and the expression of p62 was detected by western blotting. (F) Cells were treated with $250 \mu \mathrm{M}$ wogonoside for $24 \mathrm{~h}$ in the presence or absence of BafA1 $(10 \mathrm{nM})$, and the expression levels of LC3 and p62 were assessed by western blotting. $\beta$-actin was used as loading control. DMSO, dimethyl sulfoxide; LC3, light chain 3; AO, acridine orange; DAPI, 4',6-diamidino-2-phenylindole; BafA1, bafilomycin A.

these inhibitors, SB203580 exhibited the ability to counteract the cell killing activity of wogonoside while LY294002 showed the opposite effect (Fig. 5C). Furthermore, western blotting and flow cytometric analysis confirmed that p38 MAPK and $\mathrm{PI} 3 \mathrm{~K} / \mathrm{AKT} / \mathrm{mTOR} / \mathrm{p} 70 \mathrm{~S} 6 \mathrm{~K}$ signaling pathways were involved in wogonoside-induced apoptosis and autophagy (Fig. 5D-H). Taken together, these results indicate that autophagy and apoptosis induction by wogonoside were mediated partly through the p38 MAPK and PI3K/AKT/mTOR/p70S6K signaling pathways.

ROS is involved in wogonoside-induced autophagy and apoptosis. Recently, ROS were reported to be associated with autophagy and apoptosis induced by antineoplastic drugs $(19,20)$. Similarly, we found that pretreatment of cells with the antioxidant NAC rescued wogonoside-induced cell death, apoptosis and autophagy (Fig. 6A and B). All together, these results indicate that ROS are mediators between wogonoside and autophagy and apoptosis.

Wogonoside induces autophagy-related apoptosis in U87MG glioblastoma cells. To prove that the apoptosis and autophagy induced by wogonoside were not cell-specific, we examined the anticancer effect of wogonoside on another human glioblastoma cell line, U87MG. We found that wogonoside induced both apoptosis and autophagy in the U87MG cells (Fig. 7A and B). Z-VAD, 3-MA and knockdown of Beclin 1 rescued the cell death induced by wogonoside (Fig. 7C). Wogonoside also activated p38 MAPK and inhibited AKT (Fig. 7D). Furthermore, SB203580 and NAC reduced wogonoside-triggered cell death while LY294002 aggravated it (Fig. 7E). Similar results from these two glioblastoma cell lines indicated that autophagy induced by wogonoside promoted apoptosis, and that wogonoside-induced autophagy and apoptosis were due 
A

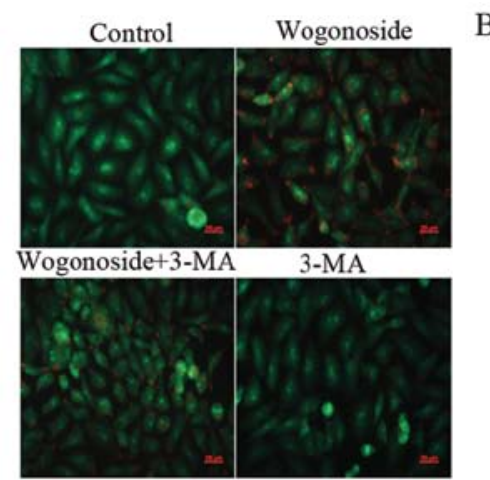

D

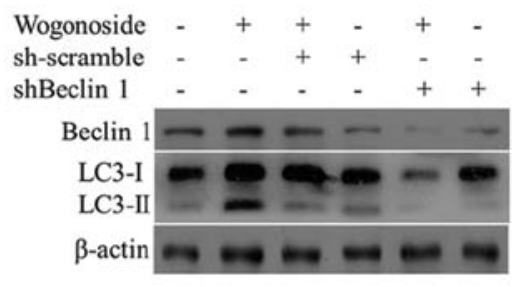

B

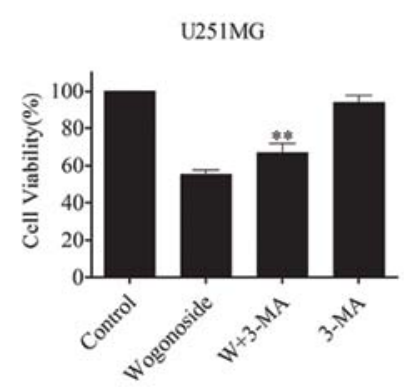

E

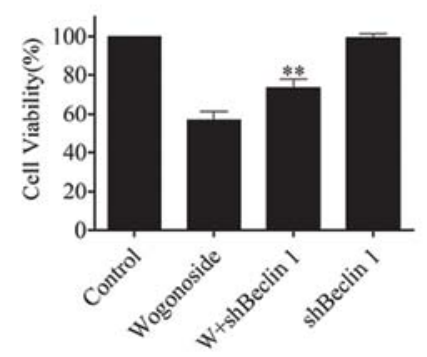

$\mathrm{C}$

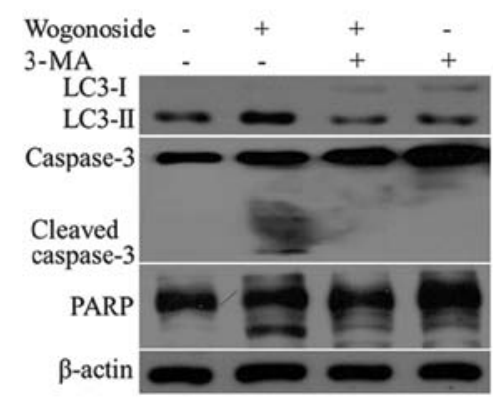

$\mathrm{F}$

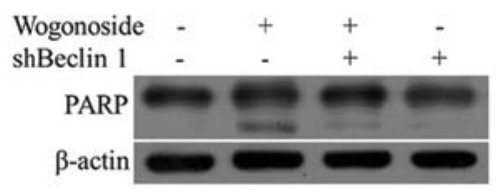

G
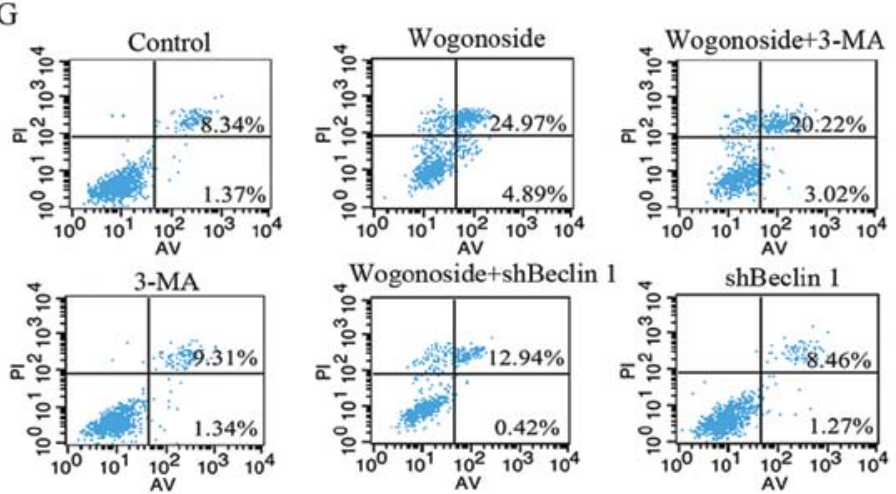

Figure 4. Inhibition of autophagy attenuates the anticancer effects of wogonoside on U251MG cells. (A) Cells were exposed to either $250 \mu \mathrm{M}$ wogonoside or $3 \mathrm{mM}$ 3-MA, or a combined treatment of wogonoside and 3-MA for $24 \mathrm{~h}$. After treatment, cells were stained with acridine orange for 15 min and observed under a fluorescence microscope. Scale bar, $20 \mu \mathrm{m}$. (B and C) Cells were treated as described above, and the cell viability (B) and the expression levels of LC3, caspase-3 and PARP (D) were analyzed. Columns; mean of three independent experiments; bars; SD. ** P<0.01 vs. cells treated with wogonoside alone. (D) Cells were transfected with either Beclin 1 shRNA or a non-target control shRNA for $72 \mathrm{~h}$ and then co-treated with $250 \mu \mathrm{M}$ wogonoside. Expression of Beclin 1 and LC3 was verified by western blotting. (E and F) Seventy-two hours after transfection, cells were treated with $250 \mu \mathrm{M}$ wogonoside for another $24 \mathrm{~h}$ and the cell viability (E) and cleavage of PARP (F) were analyzed. Columns; mean of three independent experiments; bars; SD. ${ }^{* *} \mathrm{P}<0.01 \mathrm{vs.} \mathrm{cells} \mathrm{treated}$ with wogonoside alone. $\beta$-actin was used as a loading control. (G) Cells were treated as described in A and D, and apoptosis was analyzed by flow cytometry following Annexin V/PI staining. 3-MA, 3-methyladenine; LC3, light chain 3.

to the activation of the p38 MAPK signaling pathway, inhibition of the PI3K/AKT/mTOR/p70S6K signaling pathway and generation of ROS.

\section{Discussion}

Recently, various traditional Chinese medicines have been found to exhibit promising anticancer activity (21-23). Wogonoside as an herbal medicine has shown broad anticancer effects both in vitro and in vivo. However, no studies have investigated its application in glioblastoma. The findings described herein demonstrated that wogonoside induced autophagy and apoptosis in human glioblastoma cells.

Previous studies have shown that wogonoside inhibited cell viability in breast cancer and acute myeloid leukemia (AML) cells $(7,9)$. However, the precise mechanisms under- lying wogonoside-mediated cytotoxicity have not been documented. In the present study, wogonoside displayed obvious mitochondrial apoptotic features, including the plasma membrane staining of Annexin V, caspase-3 and PARP cleavage and cytochrome $c$ translocation. Additionally, the wogonoside-mediated cytotoxicity was prevented by Z-VAD, further confirming the induction of cell death dependent of the caspase-mediated apoptotic pathways. In addition to its cytotoxicity in glioblastoma cells, we reported another function of wogonoside, the induction of autophagy. The autophagic effect of wogonoside was shown by the increase in punctate LC3 and morphologic changes. Western blotting also showed a marked increase in the expression of autophagy-related proteins. Moreover, wogonoside induced AVO formation, p62 degradation and LC3 turnover demonstrating the ability of wogonoside to elevate autophagic flux in glioblastoma cells. 
A

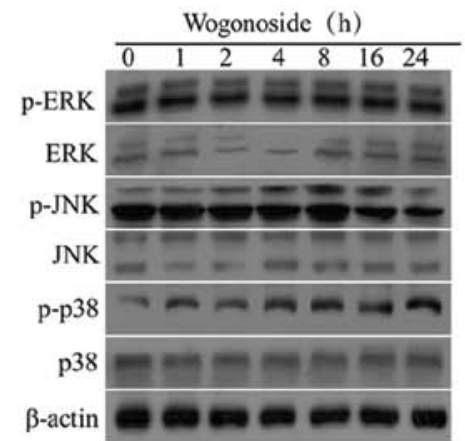

D

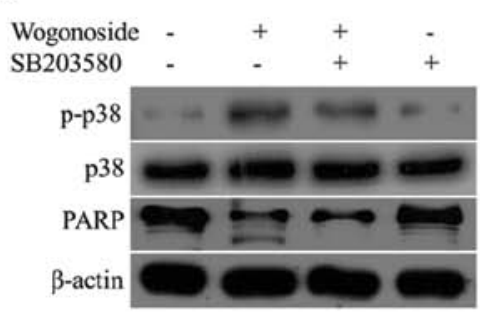

G

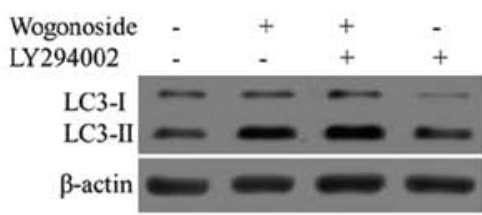

B

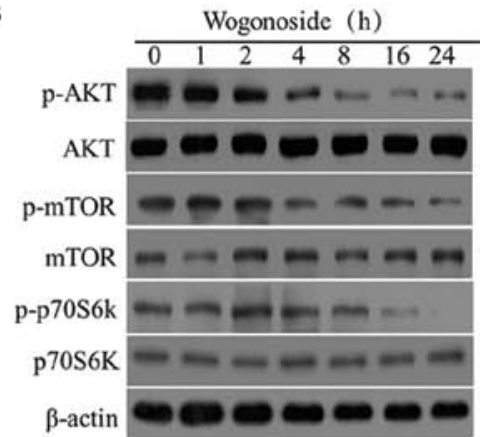

E

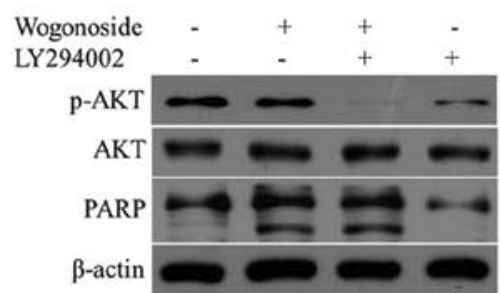

C
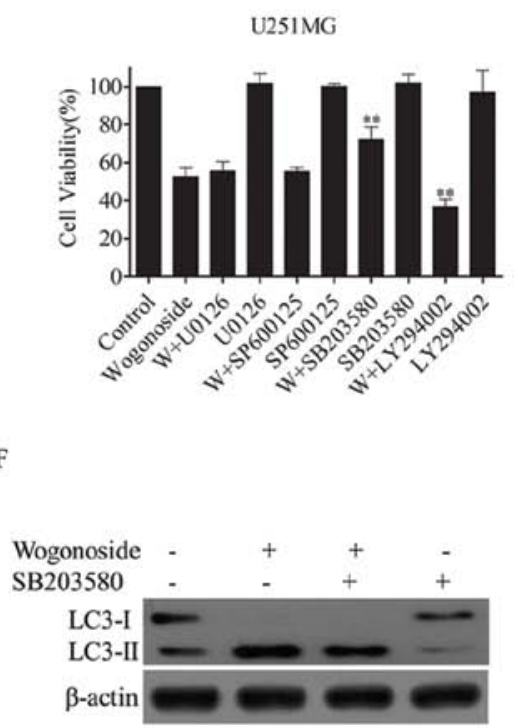

$\mathrm{H}$
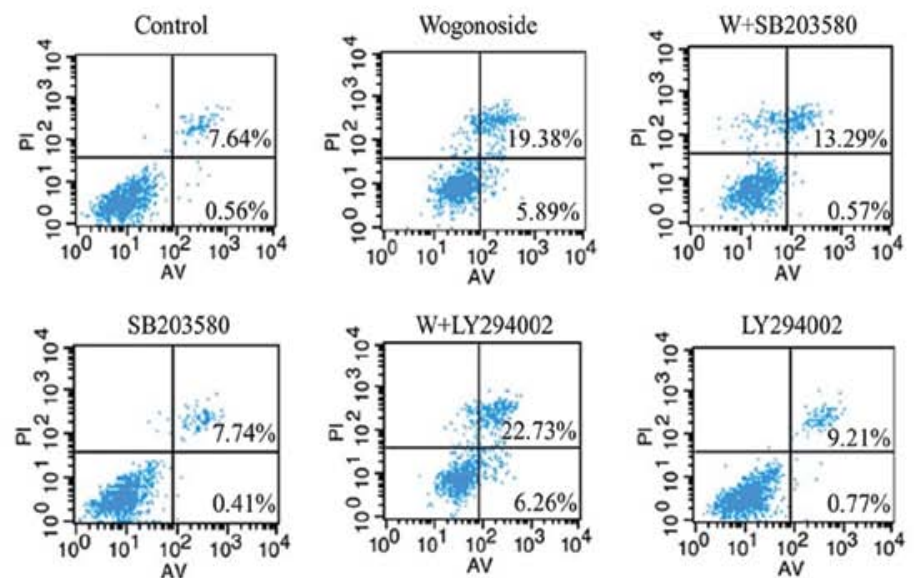

Figure 5. Effects of wogonoside on the MAPK and PI3K/AKT/mTOR/p70S6K signaling pathways in U251MG cells. (A and B) Cells were exposed to $250 \mu \mathrm{M}$ wogonoside for different time periods, and the expression levels of phosporylated (p)-ERK, ERK, p-JNK, JNK, p-p38, p38, p-AKT, AKT, p-mTOR, mTOR, p-p70S6K and p70S6K were detected by western blotting. (C) Cells were pretreated with $10 \mu \mathrm{M}$ U0126, $10 \mu \mathrm{M}$ SP600125, $10 \mu \mathrm{M}$ SB203580 and $10 \mu \mathrm{M}$ LY294002 for $1 \mathrm{~h}$ followed by treatment with $250 \mu \mathrm{M}$ wogonoside for another $24 \mathrm{~h}$. The cell viability was measured by CCK-8 assay. Columns; mean of three independent experiments; bars; SD. ${ }^{* *} \mathrm{P}<0.01$ vs. cells treated with wogonoside alone. (D, E and H) Cells were pretreated with $10 \mu \mathrm{M} \mathrm{SB} 203580$ and $10 \mu \mathrm{M}$ LY294002 for $1 \mathrm{~h}$ followed by treatment of $250 \mu \mathrm{M}$ wogonoside for another $24 \mathrm{~h}$. Apoptosis was analyzed by cleavage of PARP (D and E) and flow cytometry following Annexin V/PI staining (H). (F and G) Cells were treated as described above. The expression of LC3 was determined by western blotting. $\beta$-actin was used as a loading control. MAPK, mitogen-activated protein kinase; LC3, light chain 3.

A

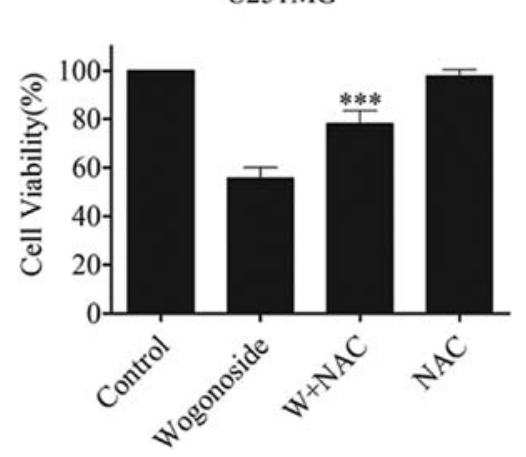

B

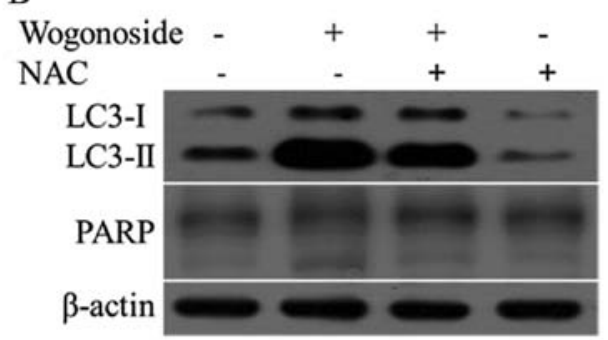

Figure 6. Wogonoside induces apoptosis and autophagy via activation of ROS in U251MG cells. (A) Cells were pretreated with $10 \mathrm{mM} \mathrm{NAC}$ for $1 \mathrm{~h}$ followed by treatment of $250 \mu \mathrm{M}$ wogonoside for another $24 \mathrm{~h}$. Cell viability was analyzed by the CCK-8 assay. Columns, mean of three independent experiments; bars, SD. ${ }^{* * * *} \mathrm{P}<0.001$ vs. cells treated with wogonoside alone. (B) Cells were treated as described above. The expression of LC3 and PARP was determined by western blotting. $\beta$-actin was used as a loading control. NAC, N-acetyl-cysteine; LC3, light chain 3. 


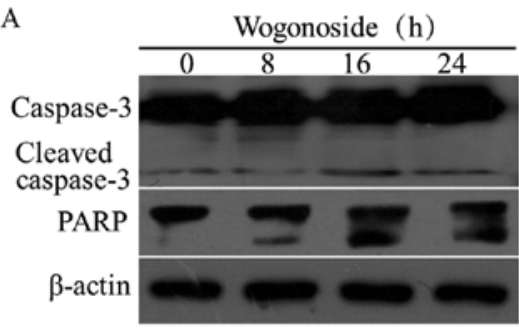

C

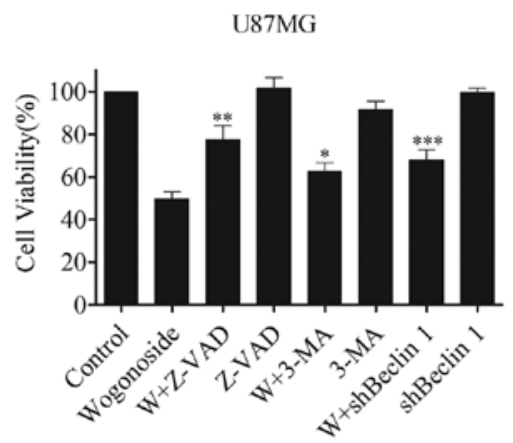

B

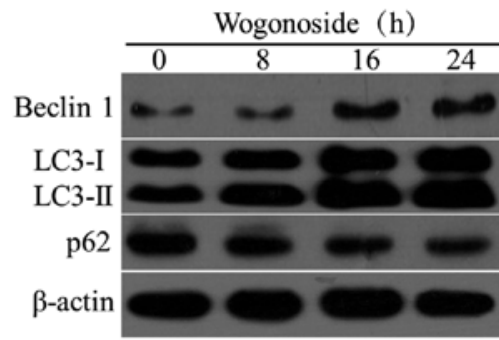

$\mathrm{D}$

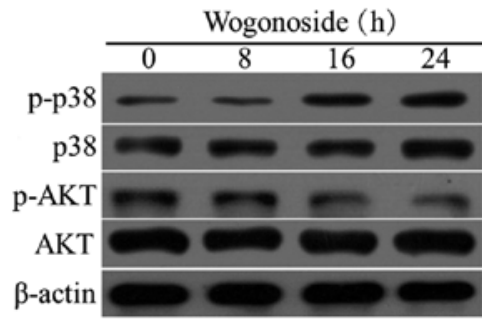

$\mathrm{E}$

U87MG

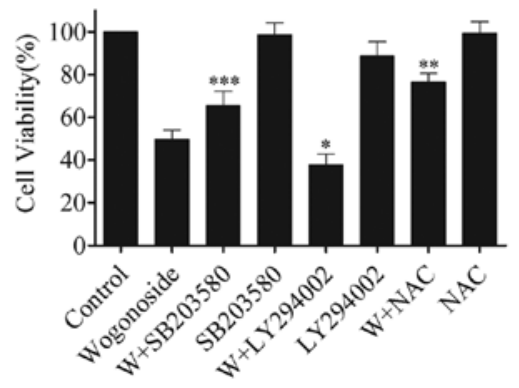

Figure 7. Anticancer effects of wogonoside on U87MG cells. (A and B) Cells were treated with $300 \mu \mathrm{M}$ wogonoside for $0,8,16$ and $24 \mathrm{~h}$, and the cell lysates were subjected to western blotting with specific antibodies. (C) Cells were exposed to either $300 \mu \mathrm{M}$ wogonoside, $25 \mu \mathrm{M} \mathrm{Z-VAD,} 3 \mathrm{mM}$ 3-MA, shBeclin 1, or a combined treatment of wogonoside and Z-VAD-FMK, 3-MA or shBeclin 1 for $24 \mathrm{~h}$, and the cell viability was measured by the CCK-8 assay. Columns, mean of three independent experiments; bars, SD. ${ }^{*} \mathrm{P}<0.05,{ }^{* *} \mathrm{P}<0.01,{ }^{* * *} \mathrm{P}<0.001$ vs. cells treated with wogonoside alone. (D) Cells were treated with $300 \mu \mathrm{M}$ wogonoside for various time periods, and the expression of $\mathrm{p} 38$ and AKT was determined by western blotting. $\beta$-actin was used as a loading control. (E) Cells were pretreated with $10 \mu \mathrm{M} \mathrm{SB} 203580,10 \mu \mathrm{M} \mathrm{LY} 294002$ or $10 \mathrm{mM} \mathrm{NAC}$ for $1 \mathrm{~h}$ followed by the treatment of $300 \mu \mathrm{M}$ wogonoside for another $24 \mathrm{~h}$, and the cell viability was measured by the CCK- 8 assay. Columns, mean of three independent experiments; bars, $\mathrm{SD}$. ${ }^{*} \mathrm{P}<0.05,{ }^{* *} \mathrm{P}<0.01,{ }^{* * * *} \mathrm{P}<0.001$ vs. cells treated with wogonoside alone. 3-MA, 3-methyladenine; NAC, N-acetyl-cysteine.

Having documented the ability of wogonoside to induce both autophagy and apoptosis, we then clarified whether the autophagy induced by wogonoside was a protective mechanism or a process leading to apoptosis. Generally, the present methods used to study the relationship between autophagy and apoptosis commonly rely on i) the presence of autophagic features in apoptotic cells and ii) rescue of cell apoptosis via suppression of autophagy (24). In our study, we found that inhibition of autophagy attenuated both the cytotoxicity and apoptosis induced by wogonoside. This phenomenon was also observed in another glioblastoma cell line, U87MG, suggesting that the pro-apoptotic role of autophagy was not cell-specific. Indeed, inhibition of autophagy has been shown to reduce the anticancer effects of minocycline (25), chlorpromazine (26) and FTY720 (27). Consist with these observations, our data suggest that autophagy may serve as an additional target for adjuvant anticancer therapy.

The MAPK and PI3K/AKT/mTOR/p70S6K pathways have been reported to play an important role in autophagy and apoptosis $(14,28)$. MAPKs are serine/threonine protein kinases belonging to the CDK/MAPK/GSK3/CLK (CMGC) kinase group. They are involved in many cellular processes, including cell differentiation, growth and death (29). $\mathrm{PI} 3 \mathrm{~K} / \mathrm{AKT} / \mathrm{mTOR} / \mathrm{p} 70 \mathrm{~S} 6 \mathrm{~K}$ is another important signaling pathway involved in the regulation of cell survival and death $(30,31)$. In our study, we found that wogonoside increased the phosphorylation of p38 MAPK and inhibited the phosphorylation of AKT, mTOR and p70S6K. Moreover, the p38 kinase inhibitor SB203580 reduced the autophagy and apoptosis induced by wogonoside while the PI3K kinase inhibitor LY294002 promoted these processes. This indicates that activation of the p38 MAPK signaling pathway and inhibition of the PI3K/AKT/mTOR/p70S6K signaling pathway by wogonoside lead to the induction of autophagy and apoptosis.

ROS are chemically reactive molecules containing oxygen. Normal levels of ROS are important for cell metabolism. However, excessive ROS can cause cell death through apoptosis, necrosis and autophagy. In the present study, we found 
that the antioxidant NAC reversed the autophagy and apoptosis triggered by wogonoside, suggesting that wogonoside induced autophagy and apoptosis via the generation of ROS. Recently, some studies have indicated that ROS could affect the MAPK and PI3K/AKT/mTOR/p70S6K signaling pathways $(32,33)$. Therefore it is unclear whether the modulation effects of wogonoside on p38 MAPK and PI3K/AKT/mTOR/p70S6K signaling pathways should be attributed to the generation of ROS and further studies are needed to clarify this issue.

Taken together, our study provides initial evidence that wogonoside inhibits the viability of human glioblastoma cells by inducing autophagy and apoptosis. Inhibition of autophagy significantly attenuated the apoptosis-inducing ability. The anticancer effect of wogonoside was associated with activation of p38 MAPK, inhibition of the PI3K/AKT/mTOR/p70S6K signaling pathway and generation of ROS. These results identify wogonoside as an attractive therapeutic agent for the development of alternative treatment protocols and possibly, for combined therapy with other anticancer agents to overcome drug resistance and achieve better outcomes.

\section{Acknowledgements}

This study was supported by grants from The National Natural Science Foundation of China (nos. 81070974 and 81271377), the Jiangsu Provincial Key Subject (no. X4200722) and Jinling Hospital of Nanjing, China (no. 2010Q017).

\section{References}

1. Germano IM, Emdad L, Qadeer ZA, Binello E and Uzzaman M Embryonic stem cell (ESC)-mediated transgene delivery induces growth suppression, apoptosis and radiosensitization, and overcomes temozolomide resistance in malignant gliomas. Cancer Gene Ther 17: 664-674, 2010.

2. Davis FG, Freels S, Grutsch J, Barlas S and Brem S: Survival rates in patients with primary malignant brain tumors stratified by patient age and tumor histological type: an analysis based on Surveillance, Epidemiology, and End Results (SEER) data, 1973-1991. J Neurosurg 88: 1-10, 1998.

3. Shrieve DC, Alexander E III, Black PM, et al: Treatment of patients with primary glioblastoma multiforme with standard postoperative radiotherapy and radiosurgical boost: prognostic factors and long-term outcome. J Neurosurg 90: 72-77, 1999.

4. Yang YZ, Tang YZ and Liu YH: Wogonoside displays anti-inflammatory effects through modulating inflammatory mediator expression using RAW264.7 cells. J Ethnopharmacol 148 271-276, 2013

5. Hui KM, Huen MS, Wang HY, et al: Anxiolytic effect of wogonin, a benzodiazepine receptor ligand isolated from Scutellaria baicalensis Georgi. Biochem Pharmacol 64: 1415-1424, 2002.

6. Li C, Lin G and Zuo Z: Pharmacological effects and pharmacokinetics properties of Radix Scutellariae and its bioactive flavones. Biopharm Drug Dispos 32: 427-445, 2011.

7. Sun Y, Zou M, Hu C, et al: Wogonoside induces autophagy in MDA-MB-231 cells by regulating MAPK-mTOR pathway. Food Chem Toxicol 51: 53-60, 2013.

8. Ikemoto S, Sugimura K, Yoshida N, et al: Antitumor effects of Scutellariae radix and its components baicalein, baicalin, and wogonin on bladder cancer cell lines. Urology 55: 951-955, 2000.

9. Chen Y, Hui H, Yang H, et al: Wogonoside induces cell cycle arrest and differentiation by affecting expression and subcellular localization of PLSCR1 in AML cells. Blood 121: 3682-3691, 2013.

10. Baumann S, Fas SC, Giaisi M, et al: Wogonin preferentially kills malignant lymphocytes and suppresses T-cell tumor growth by inducing PLCgamma1- and $\mathrm{Ca}^{2+}$-dependent apoptosis. Blood 111: 2354-2363, 2008.
11. Susin SA, Daugas E, Ravagnan L, et al: Two distinct pathways leading to nuclear apoptosis. J Exp Med 192: 571-580, 2000.

12. Wirawan E, Vanden Berghe T, Lippens S, Agostinis P and Vandenabeele P: Autophagy: for better or for worse. Cell Res 22: 43-61, 2012.

13. Codogno P and Meijer AJ: Autophagy and signaling: their role in cell survival and cell death. Cell Death Differ 12: 1509-1518, 2005.

14. Liu J, Zhang Y, Qu J, et al: $\beta$-Elemene-induced autophagy protects human gastric cancer cells from undergoing apoptosis. BMC Cancer 11: 183, 2011.

15. Trejo-Solis C, Jimenez-Farfan D, Rodriguez-Enriquez S, et al: Copper compound induces autophagy and apoptosis of glioma cells by reactive oxygen species and JNK activation. BMC Cancer 12: 156, 2012.

16. Le XF, Mao W, Lu Z, Carter BZ and Bast RC Jr: Dasatinib induces autophagic cell death in human ovarian cancer. Cancer 116: 4980-4990, 2010.

17. Chen KL, Chang WS, Cheung $\mathrm{CH}$, et al: Targeting cathepsin $\mathrm{S}$ induces tumor cell autophagy via the EGFR-ERK signaling pathway. Cancer Lett 317: 89-98, 2012.

18. Zhao Y, Luo P, Guo Q, et al: Interactions between SIRT1 and MAPK/ERK regulate neuronal apoptosis induced by traumatic brain injury in vitro and in vivo. Exp Neurol 237: 489-498, 2012.

19. Mukhopadhyay S, Panda PK, Behera B, et al: In vitro and in vivo antitumor effects of Peanut agglutinin through induction of apoptotic and autophagic cell death. Food Chem Toxicol 64: 369-377, 2014

20. Liu WJ, Luo MN, Tan J, et al: Autophagy activation reduces renal tubular injury induced by urinary proteins. Autophagy 10 : 243-256, 2014

21. Yan Y, Hao Y, Hu S, Chen X and Bai X: Hollow fibre cell fishing with high performance liquid chromatography for screening bioactive anthraquinones from traditional Chinese medicines. J Chromatogr A 1322: 8-17, 2013.

22. Shen A, Lin J, Chen Y, et al: Pien Tze Huang inhibits tumor angiogenesis in a mouse model of colorectal cancer via suppression of multiple cellular pathways. Oncol Rep 30: 1701-1706, 2013.

23. Li C, Hashimi SM, Cao S, et al: The mechanisms of Chansu in inducing efficient apoptosis in colon cancer cells. Evid Based Complement Alternat Med 2013: 849054, 2013.

24. Maiuri MC, Zalckvar E, Kimchi A and Kroemer G: Self-eating and self-killing: crosstalk between autophagy and apoptosis. Nat Rev Mol Cell Biol 8: 741-752, 2007.

25. Liu WT, Lin CH, Hsiao M and Gean PW: Minocycline inhibits the growth of glioma by inducing autophagy. Autophagy 7: 166-175, 2011.

26. Shin SY, Lee KS, Choi YK, et al: The antipsychotic agent chlorpromazine induces autophagic cell death by inhibiting the Akt/mTOR pathway in human U-87MG glioma cells. Carcinogenesis 34: 2080-2089, 2013.

27. Liao A, Hu R, Zhao Q, et al: Autophagy induced by FTY720 promotes apoptosis in U266 cells. Eur J Pharm Sci 45: 600-605, 2012.

28. Sui X, Kong N, Ye L, et al: p38 and JNK MAPK pathways control the balance of apoptosis and autophagy in response to chemotherapeutic agents. Cancer Lett 344: 174-179, 2014.

29. Pearson G, Robinson F, Beers Gibson T, et al: Mitogen-activated protein (MAP) kinase pathways: regulation and physiological functions. Endocr Rev 22: 153-183, 2001.

30. Borders EB, Bivona C and Medina PJ: Mammalian target of rapamycin: biological function and target for novel anticancer agents. Am J Health Syst Pharm 67: 2095-2106, 2010.

31. Yap TA, Garrett MD, Walton MI, Raynaud F, de Bono JS and Workman P: Targeting the PI3K-AKT-mTOR pathway: progress, pitfalls, and promises. Curr Opin Pharmacol 8: 393-412, 2008.

32. Duan WJ, Li QS, Xia MY, Tashiro S, Onodera S and Ikejima T: Silibinin activated p53 and induced autophagic death in human fibrosarcoma HT1080 cells via reactive oxygen species-p38 and c-Jun N-terminal kinase pathways. Biol Pharm Bull 34: 47-53, 2011.

33. Liu B, Cheng Y, Zhang B, Bian HJ and Bao JK: Polygonatum cyrtonema lectin induces apoptosis and autophagy in human melanoma A375 cells through a mitochondria-mediated ROS-p38-p53 pathway. Cancer Lett 275: 54-60, 2009. 